\title{
Performance Evaluation of Antenna Diversity Schemes on Mobile WiMAX Systems
}

\author{
André M. Cavalcante, Juliano J. Bazzo, Marco J. Sousa, André N. Barreto, Vicente A. de Sousa Jr. and \\ Jani Moilanen
}

\begin{abstract}
Antenna diversity is a well-known technique to increase data rate and coverage in fading environments, and it can be applied both at the transmitter or at the receiver side of a transmission link. New broadband wireless systems, such as Mobile WiMAX (IEEE 802.16e-2005 standard), rely on several antenna diversity approaches like Maximum Ratio Combining (MRC), Interference Rejection Combining (IRC) and SpaceTime-Code (STC) in order to improve the quality and efficiency of the radio access. However, inclusion of multiple antennas in a system must take into account the higher hardware investment, complexity and its impact on network optimization. In this context, this paper aims at a comparative performance evaluation of MRC, IRC and STC diversity schemes employed in mobile WiMAX systems. We focus on a simulator-based system-level analysis in order to assess the network capacity gains achievable with antenna diversity.
\end{abstract}

Keywords-antenna diversity schemes, MRC, IRC, STC

\section{INTRODUCTION}

Among the contending technologies for the next generation of mobile broadband wireless systems, WiMAX certainly deserves some special attention. WiMAX, which is based on the IEEE 802.16e-2005 standard [1], promises to deliver highspeed data transfer over a wireless link for wide-area networks in favor of an enriched user experience when using wireless services. Besides the attention devoted by IEEE, WiMAX network is also being promoted by an international industry consortium [2], whose goal is to accelerate the introduction of the system into the market by means standards-based, interoperable certificated products, namely, WiMAX Forum Certified ${ }^{\mathrm{TM}}$ products.

In present and future wireless communication systems, like WiMAX, maximising spectral efficiency is one of the most important system design goals, because radio resources are scarce and data rate requirements keep growing. A way of increasing spectral efficiency is to use multiple antennas at the transmitter and at the receiver. Multiple-antenna techniques can be used to provide either spatial diversity and interference cancellation, to improve transmission robustness, or spatial multiplexing, to increase the transmitted data rate. There is however a tradeoff between diversity and spatial multiplexing

André M. Cavalcante, Juliano J. Bazzo and Vicente A. de Sousa Jr. are with Nokia Technology Institute (INdT), Manaus, Brazil (e-mails: $\{$ andre.cavalcante $\},\{$ juliano.bazzo $\},\{$ vicente.souza $\} @$ indt.org.br $)$. Marco J. Sousa was with INdT, Manaus, Brazil, he is now with Federal University of Pará (UFPA), Belém, Brazil (e-mail: marcojsousa@gmail.com). André N. Barreto is with INdT, Brasília, Brazil (e-mail: andre.barreto@indt.org.br). Jani Moilanen is with Nokia Siemens Networks (NSN), Espoo, Finland (email: jani.moilanen@nsn.com). This work was mainly supported by Nokia Siemens Networks (NSN) - Finland, and partially by CNPq, under grant 554047/2006-3 that depends on the targets of the network operator and this is still being an interesting research topic [3].

In Mobile WiMAX, different profiles for multiple-antenna techniques are supported. The WiMAX Forum [4] has selected two profiles for downlink (DL): (i) the space-time code (STC) proposed by Alamouti for transmit diversity [5], and (ii) a 2x2 spatial multiplexing scheme [6]. These profiles can also be used on the uplink (UL), but their implementation is optional. Spatial multiplexing is however out of the scope of this paper.

The standards define the multiple antenna techniques at the transmission side, and at the receiver side the use of antenna diversity is generally exploited by a simple linear combining of the received signals at the different antennas. Some linear combining techniques are very well known in literature, like Maximal Ratio Combining (MRC), Equal Gain Combining (EGC), Antenna Selection (AS) and Interference Rejection Combining (IRC). IRC, for instance, allows us to obtain not only spatial diversity, but also to reduce interference in some scenarios. Several works [7]-[12] present a comparison among these techniques in scenarios with different channel models.

In this context, the goal of this contribution is twofold. First, the performance of the basic receive diversity schemes (i.e., MRC and IRC) are compared to the reference case, SingleInput Single-Output (SISO), in order to discuss the benefits of spatial diversity and interference cancellation in a mobile WiMAX system. Secondly, this discussion is extended by the inclusion of STC $2 \times 2$, which is one of the Multiple-Input Multiple-Output (MIMO) options of WiMAX specifications. There have been a lot of studies considering the performance evaluation of a single transmission link. In this paper we approach the problem from a different perspective, analyzing the impact of the different receive diversity schemes over the whole network. For this purpose we have used a dynamic system-level network simulator, modeling a full network with several base stations (BS).

The layout of the paper is as follows. Section II describes the modeling of antenna diversity schemes. We start presenting the approach to calculate the Signal-to-Interference-plus-NoiseRatio (SINR) for a system with multiple antennas. After that, the SINR for each antenna diversity scheme is presented. The simulation environment, used models and key assumptions are presented in section III. Performance evaluation results are presented and discussed in Section IV. Finally, the conclusions are summarized in section $\mathrm{V}$. 


\section{Antenna Diversity Modeling}

Two basic forms of antenna diversity to improve the quality of a wireless link are receive and transmit diversities. In this paper, the Maximum Ratio Combining (MRC) and Interference Rejection Combining (IRC) are evaluated for receive diversity, whereas STC (Space-Time Code) and STC with IRC (STC+IRC) are employed for transmit/receive diversities.

As mentioned before, the WiMAX performance is assessed based on system-level simulations. Instead of modeling pointto-point bit transmissions in detail, the behavior of each antenna diversity scheme is represented by different models of the post-combining SINR (Signal-to-Interference-plus-NoiseRatio) per tone, as described in the following paragraphs.

Let $x$ be the transmitted symbol at any given tone. Considering a system with one transmit antenna and $L$ receive antennas, the received signal is

$$
\mathbf{r}=\mathbf{h} x+\mathbf{n}
$$

$\mathbf{h}=\left[h_{0}, h_{1}, \ldots, h_{L-1}\right]^{T}$ is the desired user channel vector, where $h_{l}=\left|h_{l}\right| e^{j 2 \pi \theta_{l}}$ is the complex channel gain at the receive antenna $l . \mathbf{n}=\left[n_{0}, n_{1}, \ldots, n_{L-1}\right]^{T}$ is the noise-plusinterference vector, where each component $n_{l}$ has variance $\rho_{l}^{2}$.

The noise-plus-interference component is given by

$$
n_{l}=\nu_{l}+\sum_{m=1}^{M} \sqrt{P_{m}} h_{l, m}
$$

where $\nu_{l}$ is the thermal noise component with variance $\sigma_{\nu}^{2}$, which is equal for all antennas, $M$ is the number of interferers, $P_{m}$ is the average power of the $m$-th interferer and $h_{l, m}$ is the channel gain at receive antenna $l$ from the $m$-th interferer.

A generalized combiner may be represented as

$$
y=\mathbf{w}^{T} \mathbf{r}=\mathbf{w}^{T} \mathbf{h} x+\mathbf{w}^{T} \mathbf{n},
$$

where $\mathbf{w}^{T}$ is a weight vector, which depends on the chosen combining technique.

The signal-to-interference-plus-noise-ratio (SINR) is given by

$$
\gamma=\frac{E\left[\mathbf{w}^{T} \mathbf{h} \mathbf{h}^{H} \mathbf{w}^{*}|x|^{2}\right]}{E\left[\mathbf{w}^{T} \mathbf{n} \mathbf{n}^{H} \mathbf{w}^{*}\right]}=\frac{\mathbf{w}^{T} \mathbf{h} \mathbf{h}^{H} \mathbf{w}^{*} P_{0}}{\mathbf{w}^{T} \mathbf{R} \mathbf{w}^{*}},
$$

where $P_{0}$ is the average power of the desired user and $\mathbf{R}=E\left[\mathbf{n n}^{H}\right]$ is the noise-plus-interference covariance matrix.

For two receive antennas the covariance matrix is given by

$$
\mathbf{R}=\left[\begin{array}{cc}
\rho_{1}^{2} & \rho_{1,2} \\
\rho_{1,2}^{*} & \rho_{2}^{2}
\end{array}\right]
$$

where

$$
\rho_{l}^{2}=\sigma_{\nu}^{2}+\sum_{m=1}^{M} P_{m}\left|h_{l, m}\right|^{2}
$$

and

$$
\rho_{1,2}=\sum_{m=1}^{M} P_{m} h_{1, m} h_{2, m}^{*}
$$

\section{A. Interference Rejection Combining (IRC)}

The so-called interference rejection combiner (IRC) is the optimum linear combiner in terms of MMSE (Minimum Mean Square Error), which is given by the weight vector [12]

$$
\mathbf{w}_{I R C}^{T}=\mathbf{h}^{H} \mathbf{R}^{-1} \text {. }
$$

Substituting (8) in (4), the SINR of IRC can be written as

$$
\gamma_{I R C}=\mathbf{h}^{H} \mathbf{R}^{-1} \mathbf{h} P_{0} .
$$

\section{B. Maximal Ratio Combining (MRC)}

It is not always feasible to estimate the covariance among the different antennas, which is needed for IRC. This is therefore an opportunity for simpler schemes such as MRC [7]. The weight vector of MRC scheme can be obtained by assuming a diagonal covariance matrix, i.e.,

$$
\mathbf{w}_{M R C}^{T}=\mathbf{h}^{H} \mathbf{R}_{D}^{-1}
$$

where

$$
\mathbf{R}_{D}=\left[\begin{array}{cc}
\rho_{1}^{2} & 0 \\
0 & \rho_{2}^{2}
\end{array}\right],
$$

is a matrix containing only the diagonal elements of $\mathbf{R}$. The SINR of MRC scheme is given by

$$
\gamma_{M R C}=\frac{\mathbf{h}^{H} \mathbf{R}_{D}^{-1} \mathbf{h h}^{H}\left(\mathbf{R}_{D}^{-1}\right)^{H} \mathbf{h} P_{0}}{\mathbf{h}^{H} \mathbf{R}_{D}^{-1} \mathbf{R}\left(\mathbf{R}_{D}^{-1}\right)^{H} \mathbf{h}} .
$$

If the matrix $\mathbf{R}$ is diagonal in (8), i.e. if the noise is uncorrelated at the different antennas, then IRC is equal to MRC. In this case the SINR is equal to the sum of SINRs at the $L$ branches.

\section{Space-Time Code (STC)}

The first multiple antenna profile defined in WiMAX standard is the STC scheme proposed by Alamouti [5] for transmit diversity. In the IEEE 802.16e-2005 specifications, this scheme is referred to as Matrix A. Originally, Alamouti's STC was proposed to avoid the use of receive diversity on the downlink and keep the subscriber stations simple. In OFDMA-based WiMAX systems, this technique is applied in a subcarrier basis and can be described as follows.

Considering two transmit antennas, suppose we wish to transmit the symbols $s_{0}$, and $s_{1}$ in a given sub-carrier over two OFDM symbols. They can be given in the vector form $s=\left[\begin{array}{ll}s_{0} & s_{1}\end{array}\right]^{T}$. The transmitted signal over two time intervals can be represented by the matrix

$$
\mathbf{X}=\left[\begin{array}{cc}
s_{0} & s_{1} \\
-s_{1}^{*} & s_{0}^{*}
\end{array}\right]=\left[\begin{array}{ll}
x_{0,0} & x_{1,0} \\
x_{0,1} & x_{1,1}
\end{array}\right],
$$

where $x_{i, j}$ is the transmitted signal at $j$-th interval from $i$-th transmit antenna.

Considering also two receive antennas, the received signal can be represented by $\mathbf{r}_{l}=\left[\begin{array}{ll}r_{l, 0} & r_{l, 1}\end{array}\right]^{T}$, with $r_{l, i}$ representing the received signal of antenna $l$ at the time interval $i$. The channel gain among the transmit and receive antennas is represented by $\mathbf{h}_{l}=\left[\begin{array}{ll}h_{l, 0} & h_{l, 1}\end{array}\right]^{T}$, where $h_{l, j}$ is the channel 
gain between the transmit antenna $j$ and the receiver antenna $l$. Finally, $\mathbf{n}_{l}=\left[\begin{array}{ll}n_{l, 0} & n_{l, 1}\end{array}\right]^{T}$ is the noise plus interference, where $n_{l, i}$ is the component for receive antenna $l$ at time $i$.

Making $\mathbf{r}=\left[\begin{array}{ll}\mathbf{r}_{0}^{T} & \mathbf{r}_{1}^{T}\end{array}\right]^{T}, \mathbf{h}=\left[\begin{array}{ll}\mathbf{h}_{0}^{T} & \mathbf{h}_{1}^{T}\end{array}\right]^{T}$ and $\mathbf{n}=\left[\begin{array}{ll}\mathbf{n}_{0}^{T} & \mathbf{n}_{1}^{T}\end{array}\right]^{T}$, the received signal can be written as

$$
\mathbf{r}=\left[\begin{array}{cc}
\mathbf{X} & \mathbf{0} \\
\mathbf{0} & \mathbf{X}
\end{array}\right] \mathbf{h}+\mathbf{n}
$$

where $\mathbf{0}$ is a $2 \times 2$ zero matrix. This can be rewritten as

$$
\mathbf{r}^{\prime}=\mathbf{H}^{\prime} \mathbf{s}+\mathbf{n}^{\prime}
$$

where $\mathbf{r}^{\prime}=\left[\begin{array}{llll}r_{0,0} & r_{1,0} & r_{0,1}^{*} & r_{1,1}^{*}\end{array}\right]^{T}, \mathbf{H}^{\prime}=\left[\begin{array}{ll}\mathbf{h}_{0}^{\prime} & \mathbf{h}^{\prime}{ }_{1}\end{array}\right], \mathbf{h}_{0}^{\prime}=$

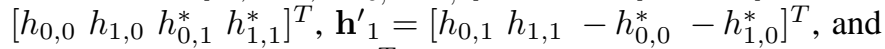
$\mathbf{n}^{\prime}=\left[\begin{array}{llll}n_{0,0} & n_{1,0} & n_{0,1}^{*} & n_{1,1}^{*}\end{array}\right]^{T}$.

Following Alamouti's original proposal, the estimated symbol ( $(\hat{\mathbf{s}})$ is

$$
\hat{\mathbf{s}}=\mathbf{H}^{\prime H} \mathbf{r}^{\prime}=\mathbf{H}^{\prime H} \mathbf{H}^{\prime} \mathbf{s}+\mathbf{H}^{\prime H} \mathbf{n}^{\prime} .
$$

The effective SINR for STC $2 \times 2$ is then given by

$$
\gamma_{S T C}=\frac{p\left(\mathbf{h}_{0}^{\prime H} \mathbf{h}_{0}^{\prime}\right)^{2}}{\mathbf{h}_{0}^{\prime H} \mathbf{R}^{\prime} \mathbf{h}_{0}^{\prime}},
$$

where $\mathbf{R}^{\prime}=E\left\{\mathbf{n}^{\prime} \mathbf{n}^{\prime H}\right\}$ and $p$ is the power per antenna. Let the noise covariance matrix among the antennas be $\mathbf{R}=$ $E\left\{\left[n_{0, j} n_{1, j}\right]^{T} \times\left[n_{0, j}^{*} n_{1, j}^{*}\right]\right\}$, then

$$
\mathbf{R}^{\prime}=\left[\begin{array}{cc}
\mathbf{R} & \mathbf{0} \\
\mathbf{0} & \mathbf{R}^{T}
\end{array}\right]
$$

This formulation was derived considering a simplified MRC combining over the two receive antennas, assuming equal noise-plus-interference variance at all receiver branches. The formulation (17) is hence optimal only if the noise variance is the same in both antennas and the noise plus interference samples are uncorrelated.

\section{STC + Interference Rejection Combining $(S T C+I R C)$}

Generally for interference scenarios, the noise-plusinterference variance is no longer equal at all receiver branches. For these cases and when the noise covariance matrix can be estimated, it is possible to use an optimum linear combiner at the STC reception equivalent to the one used for IRC, i.e.,

$$
\hat{\mathbf{s}}=\mathbf{H}^{\prime H} \mathbf{R}^{\prime-1} \mathbf{r}^{\prime}=\mathbf{H}^{\prime H} \mathbf{R}^{\prime-1} \mathbf{H}^{\prime} \mathbf{s}+\mathbf{H}^{\prime H} \mathbf{R}^{\prime-1} \mathbf{n}^{\prime}
$$

Since

$$
\mathbf{R}^{\prime-1}=\left[\begin{array}{cc}
\mathbf{R}^{-1} & \mathbf{0} \\
\mathbf{0} & \left(\mathbf{R}^{T}\right)^{-1}
\end{array}\right],
$$

the effective SINR for STC+IRC $2 \times 2$ is then

$$
\gamma_{S T C+I R C}=\frac{p\left(\mathbf{h}_{0}^{\prime H} \mathbf{R}^{\prime-1} \mathbf{h}_{0}^{\prime}\right)^{2}}{\mathbf{h}_{0}^{\prime H} \mathbf{R}^{\prime-1} \mathbf{R}^{\prime}\left(\mathbf{R}^{\prime-1}\right)^{H} \mathbf{h}_{0}^{\prime}} .
$$

\section{Wimax Simulation Model}

In order to evaluate the performance and capacity of antenna diversity schemes described above, a dynamic systemlevel simulator was used. The simulator models a standardcompliant IEEE 802.16e WiMAX multi-cell and multi-user radio network including modeling of network layout, terminal distribution and movement, radio environment, PHY layer, MAC layer (RRM and ARQ algorithms, scheduling, access schemes) and traffic generation.

\section{A. Network Topology and Deployment Scenario}

The simulator models an outdoor macrocellular topology composed of homogeneous three-sectored hexagonal cells. The cellular grid is composed of 34 base stations and 75 hexagonal sectors, as illustrated in Figure 1. Frequency reuse 1-3-1 is employed, i.e., each BS site has three sectors and all sectors are assigned the same RF channel. Table I lists the main parameters of the network topology used in the simulations.

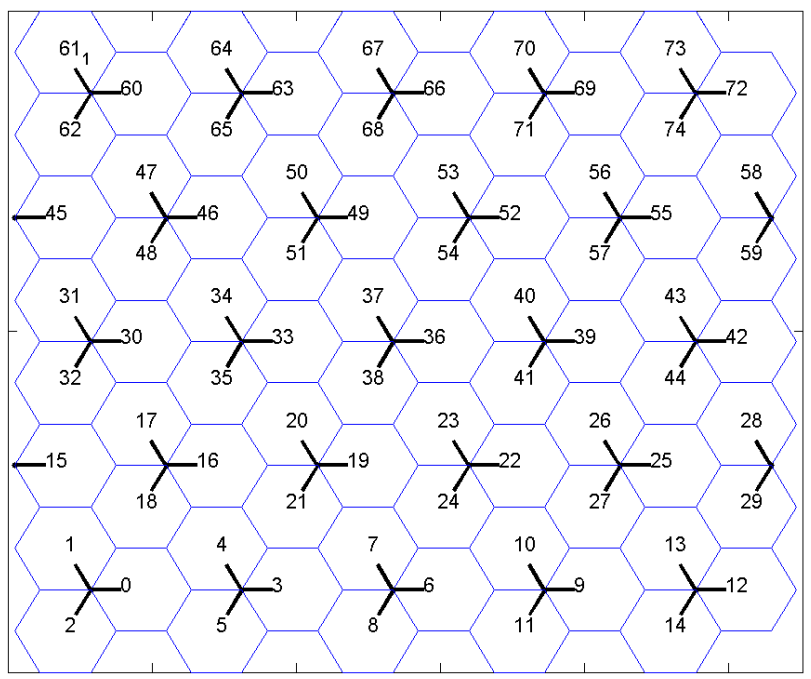

Fig. 1. Simulation Scenario

TABLE I

NETWORK TOPOLOGY AND DEPLOYMENT SCENARIO

\begin{tabular}{|l|l|}
\hline Parameters & Value \\
\hline Number of BSs & 34 \\
\hline Number of sectors per BS & 3 \\
\hline Total number of sectors & 75 \\
\hline BS-BS distance & $1.5 \mathrm{~km}$ \\
\hline Center frequency & $2.5 \mathrm{GHz}$ \\
\hline Frequency Reuse & $1-3-1$ \\
\hline Transmission Power/sector & $15 \mathrm{~W}$ \\
\hline BS height & $30 \mathrm{~m}$ \\
\hline Number of Tx antennas & $1(\mathrm{MRC}$ and IRC) and 2 (STC) \\
\hline Tx antenna pattern & $70^{\circ}(-3 \mathrm{~dB})$ with 20 dB front-to-back ratio \\
\hline Tx antenna gain & $15 \mathrm{dBi}$ \\
\hline MS height & $1.5 \mathrm{~m}$ \\
\hline Number of Rx antennas & 2 \\
\hline Rx antenna pattern & Omnidirectional \\
\hline MS Noise Figure & $8 \mathrm{~dB}$ (noise temperature $=293 \mathrm{~K})$ \\
\hline
\end{tabular}




\section{B. Propagation and Interference Models}

The results presented in this paper assume an urban macrocell environment for modeling the long-term characteristics of the wireless channel. This scenario is characterized by large cells, BS antennas above rooftop height, and high transmit power. It uses the COST-231 Hata propagation model [13] for calculating the signal path loss. Shadowing is modeled as a lognormal random variable with zero mean and standard deviation of $8.0 \mathrm{~dB}$. The shadowing correlation factor between sectors is equal to 1 for sectors of the same cell, and 0.5 for sectors of different cells.

The fading characteristics of the channel are modeled using the same model for both information-bearing and interfering signals, which ensures that the effects of multipath propagation are captured for all relevant signals in the network. The parameters of the channel model correspond to the ITU power delay profiles and the Doppler spectrum according to [14], see also Table II.

TABLE II

PROPAGATION MODELS

\begin{tabular}{|l|l|}
\hline Parameters & Value \\
\hline Path loss model & COST-231 Hata \\
\hline Lognormal shadowing & $\mu=0 \mathrm{~dB}, \sigma=8 \mathrm{~dB}$ \\
\hline Shadowing correlation & $100 \%$ inter-sector, 50\% inter-BS \\
\hline Channel model & ITU Vehicular A $(3 \mathrm{~km} / \mathrm{h})$ \\
\hline
\end{tabular}

\section{OFDMA structure, Scheduler and HARQ}

It is assumed that the mobile WiMAX TDD (Time-Division Duplex) $5 \mathrm{~ms}$ frame is divided into DL and UL subframes, with the DL subframe containing 24 (out of 47) data symbols. All BSs are assumed to be synchronized to maintain common frame start times and frame lengths, and they use the same type of permutations. Partial Usage of Subcarriers (PUSC) permutations [4] are modeled in order to take advantage of its inherent frequency diversity. For the OFDMA parameters used in the system level simulation, the reader is referred to [4].

The BS scheduler allocates the two-dimensional (timefrequency) OFDMA resources among active users. Resource allocation for the entire TDD frame is made in a RoundRobin fashion, and the modulation and coding scheme (MCS) is chosen using the available channel quality indicator (CQI) from all MSs. Hybrid Automatic Repeat Request (HARQ) is implemented using the stop-and wait protocol with multiple HARQ channels for every served MS. Chase Combining is used at the MS for successive HARQ retransmissions so that the data packets received with error are stored at the receiver and softly combined with following retransmissions. The main scheduler and HARQ parameters are presented in Table III.

\section{Simulation methodology and PHY abstraction}

The simulation model is based on the methodology recommended in [4]. Perfect time and frequency synchronization is assumed. The effect of non-ideal channel estimation is taken into account in the PHY abstraction. MSs are assigned to the best-serving BS and sector according to their downlink received signal power (considering only path loss and shadow fading). Performance statistics are collected only for users served by the BS of the center cell.

A physical layer (PHY) abstraction, based on link-level simulations, is employed to predict the PHY performance at the system-level. The PHY abstraction represents the wireless link transmission as a set of curves which describe the dependence between the channel quality indicator (CQI) and the code block error rate (BLER). In the PHY abstraction of the dynamic simulator, the SINR is calculated for all MSs at each tone for every transmission frame. Following the formulas described in Section II, the post-combined SINRs at all tones are calculated depending on the antenna diversity scheme. Then, for each code block, the SINR are mapped into a block error rate (BLER) following the EESM approach [13].

TABLE III

SCHEDULER AND HARQ PARAMETERS

\begin{tabular}{|l|l|}
\hline Parameters & Value \\
\hline Number of active users per sector & 10 \\
\hline DL/UL ratio in frame & $24 / 23$ [symbols/symbols] \\
\hline CQI feedback period/Delay & $1 / 0$ [frames/frames] \\
\hline Traffic Type & Full buffer data only \\
\hline Scheduling Algorithm & Round-Robin (RR) \\
\hline HARQ Type & Chase Combining \\
\hline Maximum number of HARQ Retransmissions & 4 \\
\hline HARQ Retransmission Delay & 1 frame \\
\hline
\end{tabular}

\section{Performance Results}

Herein, performance of antenna diversity schemes is analyzed in a specific case study. The wireless service is provided by mobile WiMAX DL in accordance with the framework and parameters presented in Section III. The SISO scheme is taken as a reference case. In our simulations, when STC is enabled we are actually transmitting with twice as much power as with a single-antenna transmission. This represents what happens in real networks, as STC is implemented by adding a new modem for each antenna. However, in an interference-limited scenario, as the one simulated here, this doesn't affect the results substantially.

Discussions are performed based on the following network statistics:

1) HARQ Packet Retransmissions: percentage of HARQ packets that were retransmitted.

2) Slot Error: slots error rate in PHY layer.

3) DL Frame Load: refers to the DL subframe utilization.

4) Spectral Efficiency: maximum achievable average throughput in terms of bits/second/Hz (PHY layer).

5) Spectral Efficiency / DL Frame Load: system spectral efficiency normalized by the DL frame load. This is needed to account for resources that are left unused because of scheduling issues.

The first two statistics are used to assess the quality of the wireless link, whereas the other are used to evaluate the performance in terms of loading and system transmission capacity.

In real-life interference-limited scenarios, interference is always spatially coloured, i.e., interference is correlated among 
TABLE IV

SIMULATION RESULTS

\begin{tabular}{|c|c|c|c|c|c|c|}
\hline $\begin{array}{c}\text { Colored } \\
\text { Interference }\end{array}$ & $\begin{array}{c}\text { Antenna Diversity } \\
\text { Scheme }\end{array}$ & $\begin{array}{c}\text { HARQ Retrans. } \\
{[\%]}\end{array}$ & $\begin{array}{c}\text { Slot Error } \\
{[\%]}\end{array}$ & $\begin{array}{r}\text { DL Frame } \\
\text { Load [\%] }\end{array}$ & $\begin{array}{c}\text { Spectral Efficiency } \\
\text { [bits/second/Hz] }\end{array}$ & $\begin{array}{c}\text { Spectral Efficiency / } \\
\text { DL Frame Load }\end{array}$ \\
\hline- & SISO & 44.84 & 49.61 & 97 & 0.2886 & 0.30 \\
\hline No & MRC & 32.40 & 31.73 & 91 & 0.6673 & 0.73 \\
\hline No & IRC & 32.40 & 31.73 & 91 & 0.6673 & 0.73 \\
\hline No & STC & 35.00 & 32.10 & 88 & 0.7077 & 0.80 \\
\hline No & STC+IRC & 33.14 & 30.55 & 88 & 0.7081 & 0.80 \\
\hline Yes & MRC & 33.71 & 33.88 & 93 & 0.6720 & 0.74 \\
\hline Yes & IRC & 29.22 & 32.72 & 90 & 0.6775 & 0.76 \\
\hline Yes & STC & 36.15 & 33.08 & 90 & 0.6952 & 0.78 \\
\hline Yes & STC+IRC & 30.49 & 28.39 & 83 & 0.7330 & 0.89 \\
\hline
\end{tabular}

different receive antennas and may have different variances. However, one of the purposes of this contribution is to verify the need for accurately modelling spatial interference correlation in system-level simulations. Therefore, the performances of antenna diversity schemes are compared for scenarios with both uncorrelated and correlated interference-plus-noise. For uncorrelated case, the interference is taken into account as an increase in the noise variance level, which is equal for all independent receive antennas.

Table IV shows the absolute values of network statistics, whereas Figures 2 and 3 show the normalized results relative to the reference case (SISO).

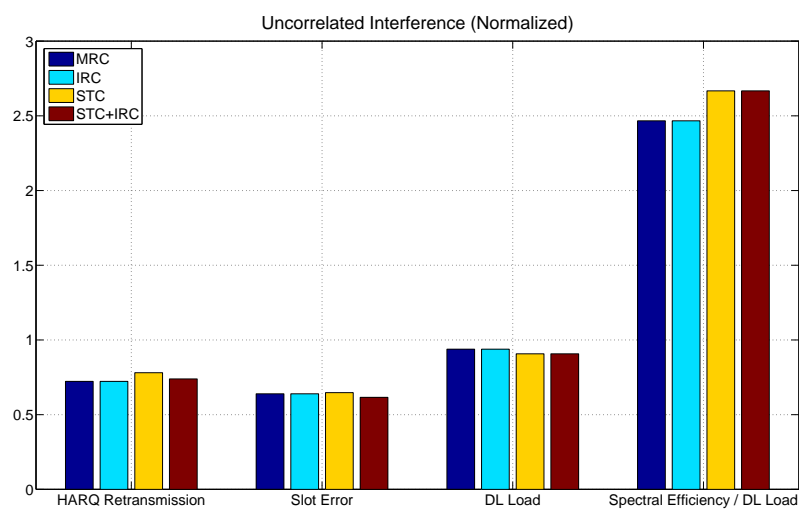

Fig. 2. Performance metrics for uncorrelated interference

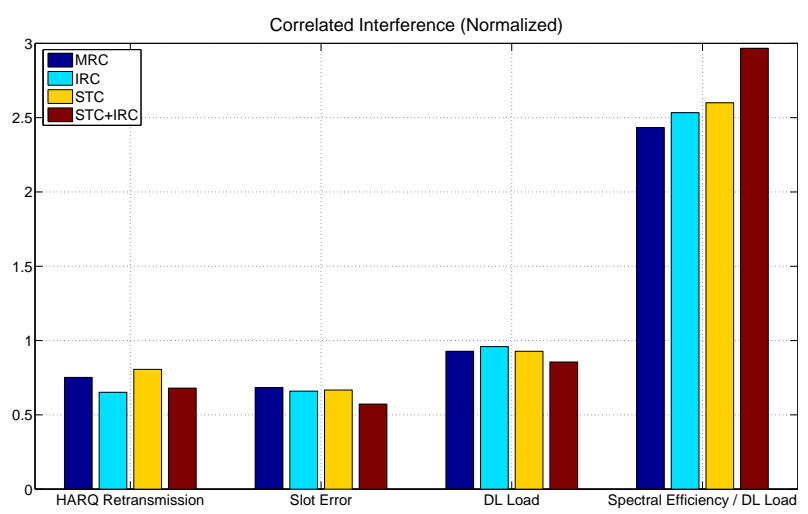

Fig. 3. Performance metrics for correlated interference
For uncorrelated interference, IRC and MRC had the same performance results for all outputs, which was already expected, because both schemes are equivalent for this case. STC outperformed MRC and IRC mainly due to the higher diversity order ( $2 \times 2$ against $1 \times 2)$.

STC+IRC and STC also present similar performance for uncorrelated interference. However, the results are not exact the same due to the small difference between the combining models at the receiver of each STC scheme. For STC, only channel gains are used to combine the signals, as mentioned in Section II-C, whereas in STC+IRC, the different variances of the noise plus interference are also considered. This also explains the performance comparison between MRC and STC. The better spectral efficiency of STC compared to MRC is due to higher order antenna diversity of STC (2x2). However, MRC outperformed STC in terms of slot error and HARQ retransmissions, because of the simplified combining model employed in STC.

When correlated interference is considered we notice quite significant differences in the performance results, particularly when IRC is considered, what justifies this more accurate modelling. IRC outperformed MRC in all statistics. This shows that IRC can favor to an improvement on metrics like effective user throughput. STC+IRC had the best performance among the schemes. The diversity provided by STC schemes together with the IRC interference-cancelling capabilities are the reasons for this result.

Assuming that the channel and the interference covariance matrix are known at the receiver, then IRC (or IRC+STC) is always better than MRC (or IRC). The gain that can be achieved with IRC is however dependent on the interference characteristics. This is usually described in terms of DIR (Dominant Interferer Ratio), which represents the ratio between the interference power of the strongest interferer and the rest of the interference and noise. Scenarios with one strong interferer, and consequently a highest DIR, lead to a high interference correlation among the antennas which can increase the gain of IRC over MRC. On the other hand, scenarios with many weak interferers result on a nearly-white interference, and, hence, the performances of IRC and MRC are nearly the same.

The performance of IRC is strongly dependent on the correct estimation of the interference covariance matrix. In our simulations we have assumed that the covariance matrix 
is perfectly known at the receiver. This estimation is attainable in the downlink with reasonable accuracy, because interference comes always from the same transmitters (BSs), and wideband reference signals are regularly broadcast, such as the preamble sent in every WiMAX TDD frame. However, in the uplink of OFDMA packet-based systems, like mobile WiMAX, pilot symbols are available only for short transmission bursts and only for some subcarriers, which may suffer interference from different sources over time. Reliable channel estimation is a difficult task under these conditions, depending on the good design of pilot sequences. Therefore, estimation errors in the noise covariance matrix can make the use of IRC unfeasible in the uplink.

In general, antenna diversity schemes can provide meaningful performance gains in a wireless communication system. An estimate of their real benefits can be obtained from the simulation results shown here, when comparing any antenna diversity scheme to the reference case (SISO). In all cases huge performance improvements are achieved.

\section{Conclusions}

A performance evaluation of MRC, IRC and STC diversity schemes for mobile WiMAX system were shown based on results collected from a dynamic WiMAX system-level simulator. As expected, the combination of STC and IRC provides a better performance in terms os spectral efficiency. However, in the real scenario, the performance is dependent of the knowledge of the covariance matrix, and matrix estimation was not analysed here. Among the other schemes, MRC presents the best complexity-benefit tradeoff for uncorrelated interference among the receive antennas. The STC+IRC presents meaningful gains in the tested case, but a study taking into account the channel estimation errors would be necessary for a better understanding of issues related to the channel estimation.

Simulation results indicate a potential performance gain if a dynamic change of diversity scheme is applied, without the dependency on covariance matrix calculation. The criteria for this dynamic selection might be subject of further studies.

\section{ACKNOWLEDGMENT}

The authors gratefully acknowledge Antti Sevon and Mika Kolehmainen for very useful discussions and support on system-level simulator development.

\section{REFERENCES}

[1] "IEEE 802.16e-2005, Part 16: Air Interface for Fixed Broadband Wireless Access Systems", Amendment for physical and MAC layers for combined fixed and mobile operation in licensed bands, 2005.

[2] www.wimaxforum.org

[3] J.G. Proakis, "Digital Communications"

[4] WiMAX Forum, “ Mobile WiMAX - Part I: A Technical Overview and Performance Evaluation", white paper, August 2006.

[5] S. M. Alamouti, "A Simple Transmit Diversity Technique for Wireless Communications," in IEEE J. Selected Areas in Communications, vol. 16, no. 8, pp. 1451 - 1458, October 1998.

[6] M. Damen, H. El Gamal, and G. Caire, "Lattice Coding and Decoding Achieve the Optimal Diversity-Multiplexing Tradeoff of MIMO," IEEE Trans. Inform. Theory, pp. 3601-3621, June 2004.
[7] T. Eng and N. Kong and L. B. Milstein, "Comparison of diversity combining techniques for Rayleigh-fading channels," in IEEE Trans. Commun., vol. 44, No. 9, Sep. 1996, pp. 1117-112

[8] L. Fang and G. Bi and A. C. Kot, "New method of performance analysis for diversity reception with correlated Rayleigh-fading signals," in IEEE Trans. Veh. Tech., vol. 49, no. 5, Sep. 2000, pp. 1807-1812

[9] Y. Ma, "Impact of correlated branches in Rician Fading Channels," in Proc. Int'l Commun. Conf. (ICC 2005), Seoul, S. Korea, May 2005

[10] G. K. Karagiannidis and D. A. Zogas and N. C. Sagias and S. A. Kotsopoulos and G. S. Tombras, "Equal-gain and maximum-ratio combining over nonidentical Weibull fading channel," in IEEE Trans. Wireless Commun., vol. 4, no. 3, May 2005, pp. 841-846

[11] B. Chun, "A hybrid selection/equal-gain combining over correlated Nakagami-m fading channels," in IEEE Commun. Letters, vol. 11, no. 2, Feb. 2007

[12] A. N. Barreto and R. D. Vieira, "A critical analysis of receiver diversity with multiple antennas with spatially coloured noise," in Proc. Workshop on Signal Processing Advances in Wireless Communications (SPAWC), Recife, Brazil, Jun. 2008

[13] IEEE 802.16m-08/004r5, "IEEE 802.16m Evaluation Methodology Document (EMD)"

[14] Recommendation ITU-R M.1225, "Guidelines for Evaluation of Radio Transmission Technologies for IMT-2000," 1997. 